\title{
Rearrangements in a hydrophobic core region mediate cAMP action in the regulatory subunit of PKA
}

\section{Claudia Hahnefeld, Daniela Moll, Maik Goette and Friedrich W. Herberg*}

Department of Biochemistry, University of Kassel, D-34132 Kassel, Germany

${ }^{*}$ Corresponding author

e-mail: herberg@uni-kassel.de

\begin{abstract}
cAMP-dependent protein kinase (PKA) forms an inactive heterotetramer of two regulatory ( $R$; with two CAMPbinding domains $A$ and $B$ each) and two catalytic (C) subunits. Upon the binding of four cAMP molecules to the $\mathrm{R}$ dimer, the monomeric $\mathrm{C}$ subunits dissociate. Based on sequence analysis of cyclic nucleotide-binding domains in prokaryotes and eukaryotes and on crystal structures of cAMP-bound $R$ subunit and cyclic nucleotide-free Epac (exchange protein directly activated by cAMP), four amino acids were identified (Leu203, Tyr229, Arg239 and Arg241) and probed for cAMP binding to the $R$ subunits and for R/C interaction. Arg239 and Arg241 (mutated to Ala and Glu) displayed no differences in the parameters investigated. In contrast, Leu203 (mutated to Ala and Trp) and Tyr229 (mutated to Ala and Thr) exhibited up to 30fold reduced binding affinity for the $C$ subunit and up to 120 -fold reduced binding affinity for cAMP. Tyr229Asp showed the most severe effects, with 350 -fold reduced affinity for CAMP and no detectable binding to the $\mathrm{C}$ subunit. Based on these results and structural data in the cAMP-binding domain, a switch mechanism via a hydrophobic core region is postulated that is comparable to an activation model proposed for Epac.
\end{abstract}

Keywords: cAMP-dependent protein kinase; exchange protein directly activated by CAMP; surface plasmon resonance.

\section{Introduction}

cAMP-dependent protein kinase (PKA) is a key enzyme in intracellular signal transduction and serves as a model system for protein kinases. The importance of PKA is being increasingly identified with regard to its involvement in several diseases. Recent examples are potential associations with the Carney complex disease (Stergiopoulos and Stratakis, 2003) and HIV; for a review see Tasken and Aandahl (2004).

PKA is inactive in its heterotetrameric form, which consists of two catalytic $(C)$ subunits and a regulatory $(R)$ subunit dimer. The biochemical properties of PKA are defined primarily by its $R$ subunits. Two general types of $\mathrm{R}$ subunits have been described, RI and RII, with two subtypes each, $\alpha$ and $\beta$. Activation of the enzyme is achieved by the cooperative binding of the second messenger cAMP to the $R$ subunits, leading to dissociation of the holoenzyme complex (Krebs and Beavo, 1979; Taylor et al., 1990; Herberg et al., 1994). The crystal structures of deletion mutants of Rl $\alpha$ (Su et al., 1995; Wu et al., 2004a,b), of RII $\beta$ (Diller et al., 2001) and of the fulllength C subunit (Knighton et al., 1991; Bossemeyer et al., 1993) are available. Recently, a high-resolution crystal structure of a truncated $R$ subunit/C subunit complex was published (Kim et al., 2005). However, the crystallization of an entire holoenzyme structure is still awaited. Thus, on the molecular level the mode of action of cAMP, starting with docking of the cyclic nucleotide to the $R$ subunits and finally resulting in release of the $C$ subunits, remains unclear.

Besides the $\mathrm{N}$-terminal dimerization domain, the $\mathrm{R}$ subunits contain an inhibitory site for $C$ subunit binding and two tandem cAMP-binding domains, $\mathrm{A}$ and $\mathrm{B}$. The general fold of the cAMP-binding domain is highly conserved in prokaryotes (for example in the catabolite gene activator protein, CAP; Weber et al., 1987), as well as in eukaryotes. Other cAMP-binding proteins in eukaryotes are cyclic nucleotide-gated (CNG) channels (for a review see Kaupp and Seifert, 2002) and the exchange protein directly activated by cAMP (Epac). Epac1 and Epac2 are guanine nucleotide exchange factors (GEFs) and activate the small GTPases Rap1 and Rap2 (de Rooij et al., 1998, 2000; Kawasaki et al., 1998), with one cyclic nucleotide monophosphate-binding domain in Epac1 and two in Epac2 (Rehmann et al., 2003). In contrast to PKA, in Epac the regulatory domain (including the cyclic nucleotide-binding domains) and the catalytic domain responsible for the guanine nucleotide exchange activity are located on a single polypeptide chain.

For PKA type I, a sequential dissociation mechanism has been proposed in which CAMP binds first to CAMPbinding site $B$, which is exposed to the solvent in the holoenzyme (Ogreid and Doskeland, 1981; Herberg et al., 1996). After conformational rearrangement, the CAMPbinding site $A$ becomes more accessible to cyclic nucleotides and binding of additional CAMP to site A triggers release of the catalytic subunit.

Based on the crystal structures of Rl $\alpha$ (Su et al., 1995) and RII $\beta$ (Diller et al., 2001), a network of ionic interactions (salt bridges) is potentially responsible for transmitting the cAMP signal to conformational changes, finally resulting in dissociation of the $\mathrm{C}$ subunit. However, sequence alignments of cAMP-binding proteins throughout the animal kingdom and bacteria demonstrate that several key residues, displaying mostly hydrophobic character, seem to be highly conserved. Based on the cyclic nucleotide-free crystal structure of Epac2, it was concluded that the interaction of hydrophobic amino acids may trigger the physiological response to cAMP binding (Rehmann et al., 2003). 


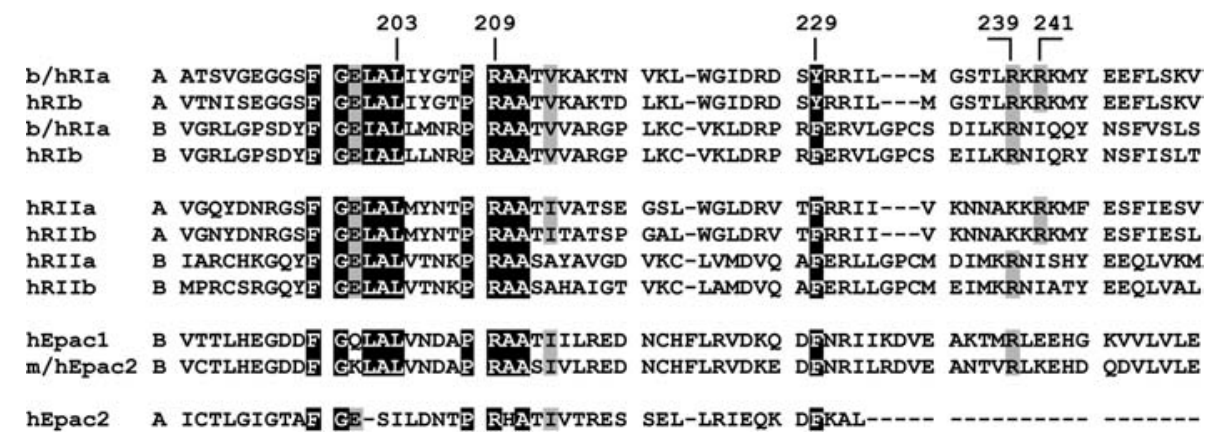

Figure 1 Amino acid sequence alignment of the cAMP-binding domains in regulatory subunits of PKA and in Epac.

The amino acid sequences used were $b / h R l \alpha$ (bovine Rl $\alpha$, NCBI Entrez protein data base accession number: OKBO1R; human Rl $\alpha$, AAB50922), hRI $\beta$ (human RI $\beta, A A C 37564$ ), hRIl $\alpha$ (human RIl $\alpha, N P \_004148$ ), hRII $\beta$ (human RII $\beta$, AAA60099), hEpac1 (human Epac1, 095398), and m/h Epac2 (murine Epac2, 1O7FA; human Epac2, NP_008954). The numbering refers to the amino acid sequence of bovine Rl $\alpha$. The alignment was performed with the web-based software DIALIGN 2.2.1 (Morgenstern, 1999). Important invariant (highlighted in black) and highly conserved (lightly shaded) residues are highlighted.

Chemical modifications and the crystal structure of a deletion protein of Rl $\alpha$ indicate that salt bridges with carboxylates in PKA are formed by positively charged residues (Buechler and Taylor, 1990; Su et al., 1995; Gibson et al., 1997). The crystal structure clearly revealed that two prominent Arg residues, Arg239 and Arg241, are positioned on a connecting helix (Symcox et al., 1994; Sorol et al., 2000). This $\alpha \mathrm{C}$-helix (residues 234-260) transmits the effects of cAMP on the $B$ domain to the $A$ domain and may also provide a direct interface for $C$ subunit interaction (Huang and Taylor, 1998). Both crucial Arg residues are highly conserved in the A domains of $\mathrm{RI}$ subunits based on sequence comparisons (Figure 1). In the $\mathrm{B}$ domains, one conserved Arg residue can be found, but cannot clearly be assigned to Arg239 or Arg241 in the A domain (Shabb and Corbin, 1992; Canaves and Taylor, 2002). Based on $\mathrm{H} /{ }^{2} \mathrm{H}$ exchange studies, Tyr229 was protected upon $\mathrm{C}$ subunit binding, suggesting that this residue is important for the interaction between $R$ and $C$ subunits (Anand et al., 2002).

Using type I $\alpha$ PKA, the mode of action of cAMP was investigated based on two different sets of mutant proteins. In a first set of mutant proteins, a model was probed in which ionic interactions were disrupted by exchange of the two conserved Arg residues. An alternative model based on a proposed lid mechanism (Rehmann et al., 2003) was tested with a second set of mutant proteins by exchanging the conserved residues Leu203 and Tyr229.

\section{Results}

A strategy combining rational protein design and biophysical/biochemical analysis served to unravel the effects of cAMP, starting from cAMP binding up to dissociation of the PKA holoenzyme. Several key amino acids, potentially involved in transmitting the cAMP signal, were exchanged in the Rl $\alpha$ subunit using site-directed mutagenesis. Since it is difficult to kinetically distinguish the binding patterns of a highly cooperatively working regulatory protein (Herberg et al., 1994, 1996), a monomeric, truncated $\mathrm{R}$ subunit was generated. This construct (bovine Rl $\alpha \Delta 1-92 \Delta 260-379$; Ringheim and
Taylor, 1990) referred to here as the R A-domain, retains both the capability to reversibly inhibit the $C$ subunit and to respond to CAMP. However, it allows investigation of $\mathrm{R} / \mathrm{C}$ subunit interaction, as well as $\mathrm{R}$ subunit/cAMP interaction, in a simplified 1:1 binding model. The R A-domain was then used as the basis for site-directed mutagenesis of several key residues. Arg239 and Arg241, highly conserved in the R subunits only (Figure 1), were mutated to Ala and Glu to investigate a mechanism based on the formation of salt bridges. This was deduced from the crystal structures of nucleotide-bound $\mathrm{Rl} \alpha$ (Su et al., 1995) and RII $\beta$ (Diller et al., 2001) and from primary sequence comparisons (Symcox et al., 1994; Sorol et al., 2000). Alternatively, to probe a mechanism based on an interaction network of hydrophobic amino acids as exemplified in the nucleotide-free crystal structure of Epac (Rehmann et al., 2003), Leu203 was mutated to Ala and Trp, and Tyr229 was replaced by Ala, Asp, Thr and Phe.

$\mathrm{R}$ A-domain mutant proteins were overexpressed in $E$. coli yielding high levels of expression. Lysis by sonification or by French press treatment resulted in almost insoluble protein. Nevertheless, it was possible to purify the proteins via affinity chromatography using 6-AHCAMP agarose. It has been demonstrated that CAMP analogs with a substitution in the $\mathrm{N}^{6}$ position of the purine ring display high affinity for the cAMP-binding site A in the full-length Rl $\alpha$ subunit (Ogreid et al., 1989). The protein could be eluted with an excess of cGMP, which was subsequently removed. Independently of the amino acid substitution introduced, all cGMP-eluted proteins displayed similar Stokes' radii as demonstrated by highresolution gel filtration, indicating that the overall shape of the mutant protein did not deviate from the $R A$ domain wild type (WT) (data not shown). Furthermore, CD measurements confirmed that the relative $\alpha$-helical content was not altered when comparing R A-domain WT and protein mutated to alanine (Figure 2; Leon et al., 2000). At $22^{\circ} \mathrm{C}$ the mutants showed practically identical CD spectra, with minima at 209 and $222 \mathrm{~nm}$. Hence, the global secondary structure of the mutant proteins was not affected severely by one of the point mutations introduced, as corroborated by CD spectra and analytical gel filtration. 


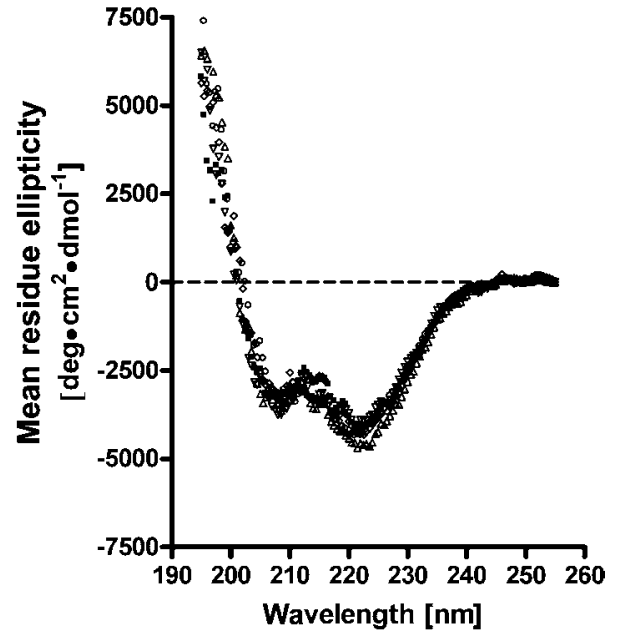

Figure 2 Circular dichroism (CD) analysis.

Circular dichroic spectra of R A domain WT ( $\square)$, R241A $(\triangle)$, Y229A ( $\nabla)$, R239A $(\diamond)$ and L203A $(\bigcirc)$. Measurements using $15 \mu \mathrm{M}$ of each protein were performed on a Jasco J-720 spectropolarimeter at $22^{\circ} \mathrm{C}$ scanning from 195 to $255 \mathrm{~nm}$. The mean residue ellipticity is shown as a function of wavelength.

To investigate the ability of a mutant $R$ subunit and a wild-type $\mathrm{C}$ subunit to form holoenzyme, both subunits were mixed and assayed for the remaining phosphotransferase activity according to Cook et al. (1982). Holoenzyme in a 1:1 stoichiometry was expected to form instantaneously, since the $\mathrm{R}$ subunits prepared were nucleotide-free (see materials and methods). Indeed, C subunit activity was immediately inhibited by the addition of R A-domain WT, reflecting holoenzyme formation (Figure 3). The functionality of the holoenzyme complex was proven by the fact that cAMP restored activity.

Hence, the affinity of the $R$ subunit for the $C$ subunit was investigated using a spectrophotometric assay. C subunit at a fixed concentration ( $30 \mathrm{~nm}$ ) was incubated with different concentrations of $\mathrm{R}$ subunit. After plotting the residual activity against the $\mathrm{R}$ subunit concentration, an $\mathrm{EC}_{50}$ value of $33 \mathrm{~nm}$ for the $\mathrm{R} A$-domain WT was extracted (Figure $4 A, B$ ). All proteins with mutations of the Arg residues (Arg239Ala/Glu, Arg241Ala/Glu) showed similar results.

In contrast, significantly higher amounts of the R Adomain Leu203 and Tyr229 mutant protein were needed to inhibit the catalytic subunit. Leu203Ala and Leu203Trp showed approximately 10 -fold higher $\mathrm{EC}_{50}$ values (330 and $380 \mathrm{nM}$, respectively); $\mathrm{R}$ A-domain Tyr229Ala $(620 \mathrm{~nm})$ displayed an even higher $\mathrm{EC}_{50}$ value (Figure 4A,B). Interestingly, mutation of Tyr229 to Phe yielded a protein with $\mathrm{C}$ subunit inhibition similar to the $\mathrm{R} A$ domain WT. The amino acid change to Phe is conserved: most cyclic nucleotide-binding domains display a Phe residue instead of Tyr (Shabb and Corbin, 1992; Rehmann et al., 2003).

Based on these results, two groups of mutant proteins could be identified: (1) the Arg239 and Arg241 mutant proteins displaying wild-type-like behavior; and (2) the Tyr229 and Leu203 mutant proteins with decreased affinity for the C subunit. The R A-domain Tyr229Thr protein (83 $\mathrm{nm}$ ) ranged between the Arg and Tyr/Leu proteins, with a three-fold enhanced $\mathrm{EC}_{50}$ value compared to the

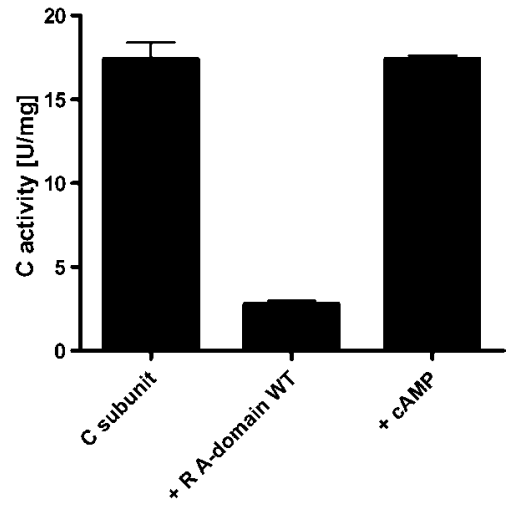

Figure 3 Catalytic activity of the $\mathrm{C} \alpha$ subunit after cAMP-mediated dissociation of the holoenzyme.

Substrate phosphorylation by $30 \mathrm{~nm} \mathrm{C} \alpha$ subunit was monitored for $1 \mathrm{~min}$ and the catalytic activity was calculated. Catalytic activity was inhibited in the presence of $37 \mathrm{nM} R$ A-domain WT and regained after addition of $50 \mu \mathrm{M}$ cAMP. For further details see materials and methods.
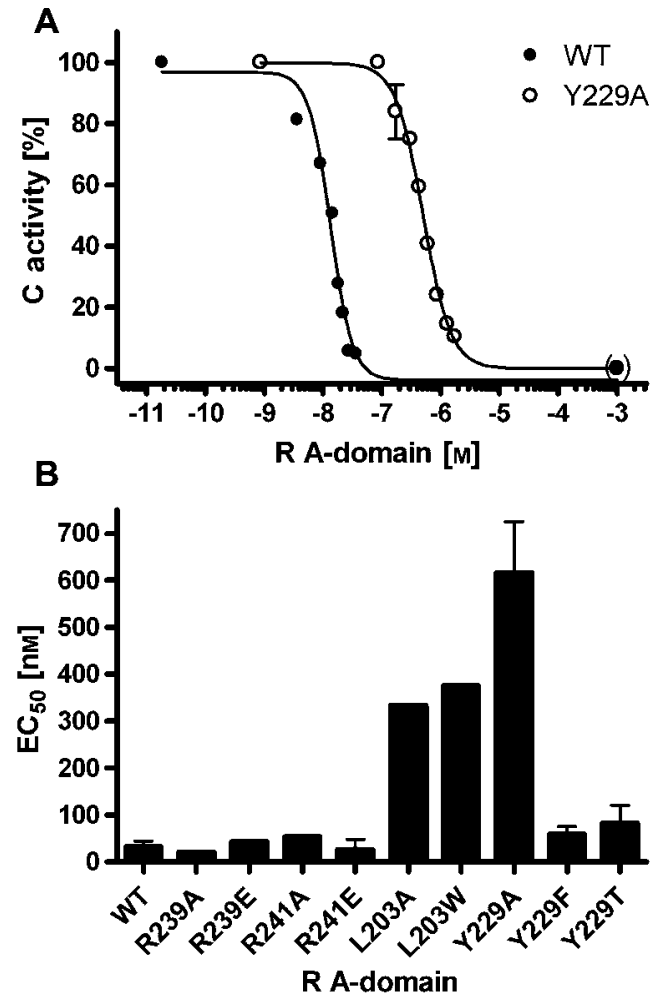

Figure 4 Holoenzyme formation of the $\mathrm{C} \alpha$ subunit with the $\mathrm{R}$ A-domain.

(A) Half-logarithmic plot of $\mathrm{R}$ subunit concentration vs. catalytic activity of the $\mathrm{C}$ subunit. The $\mathrm{EC}_{50}$ value of the sigmoidal doseresponse curve yields the $\mathrm{R}$ subunit concentration necessary to inhibit $50 \%$ of the $\mathrm{C}$ subunit activity: R A-domain WT (filled circles); R A-domain Tyr229Ala (open circles). (B) The C subunit (30 $\mathrm{nM}$ ) was preincubated with varying concentrations of R Adomain WT or mutant proteins in $100 \mu$ l of assay mix for $1 \mathrm{~min}$ at room temperature $\left(22^{\circ} \mathrm{C}\right)$ as described under materials and methods. The reaction was started by the addition of Kemptide (200 $\mu \mathrm{M}$ final concentration). The bar diagram shows the $\mathrm{R}$ subunit $\mathrm{EC}_{50}$ value derived from a sigmoidal dose-response curve as in (A). The maximum activity after addition of CAMP $(75 \mu \mathrm{M})$ remained constant in each case. 


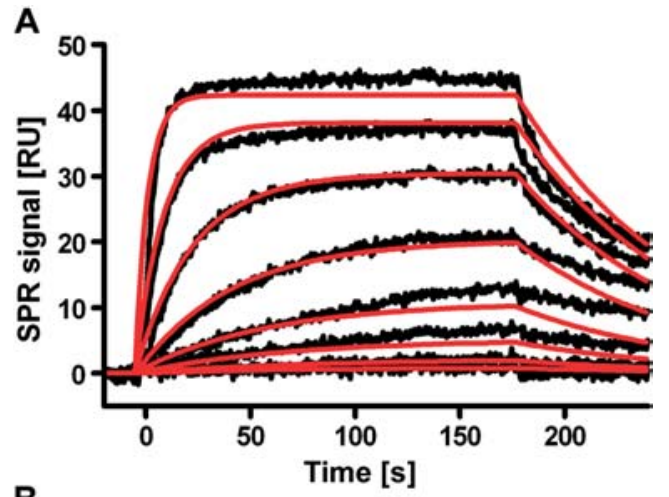

B

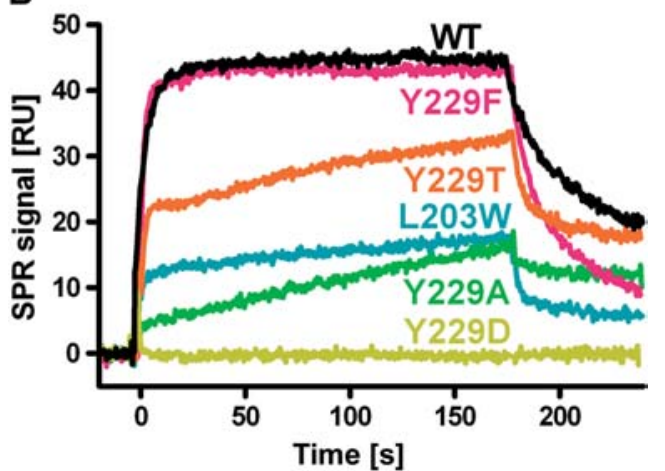

Figure 5 Surface plasmon resonance experiments for the association and dissociation behavior of the R A-domain WT on immobilized $\mathrm{C} \alpha$.

(A) The catalytic subunit was immobilized to 470 response units $(R U)$ via amine coupling on a CM5 sensor chip (Biacore $A B$ ) according to Herberg et al. (1994). Interaction of the R subunit with the immobilized $\mathrm{C}$ subunit was determined at $25^{\circ} \mathrm{C}$ in buffer C (20 mM MOPS, pH 7, $150 \mathrm{~mm} \mathrm{NaCl}, 1 \mathrm{~mm} \mathrm{MgCl}, 500 \mu \mathrm{M}$ ATP, $0.005 \%$ surfactant P20) using a Biacore 2000 instrument. Concentrations ranging from 0.7 to $400 \mathrm{nM} R$ subunit were injected at a flow rate of $20 \mu \mathrm{l} / \mathrm{min}$. Association was monitored for $3 \mathrm{~min}$ and dissociation was initiated by switching to buffer and monitored for $1 \mathrm{~min}$. The regeneration of the $C$ subunit surface was achieved by an injection of $1 \mathrm{mM}$ cAMP and $2 \mathrm{mM}$ EDTA in running buffer twice for 1 min each (not shown). The Langmuir 1:1 fits are superimposed (red). All other proteins were measured accordingly (see Table 1). (B) Binding of different R Adomain WT and mutant proteins to immobilized $\mathrm{C} \alpha$. The experimental conditions were as for (A). The binding patterns of different $R$ subunit mutants are shown at a concentration of $400 \mathrm{~nm}$ for R A-domain WT, Tyr229Asp, Tyr229Phe and 444 nM for Leu203Trp, Tyr229Ala, Tyr229Thr. The mutant protein R Adomain Tyr229Asp did not bind at all.

R A-domain WT. R A-domain Tyr229Asp did not inhibit $30 \mathrm{nM} \mathrm{C}$ subunit, even up to a concentration of $3 \mu \mathrm{M}$.

Using Biacore instruments, surface plasmon resonance was applied to measure the detailed interaction kinetics between covalently immobilized $C$ subunit (see Materials and methods) and nucleotide-free R A-domain WT and mutant proteins, respectively. Association $\left(k_{\text {ass }}\right)$ and dissociation $\left(k_{\text {diss }}\right)$ rate constants were determined separately from an individual set of binding curves using different $R$ subunit concentrations (Figure $5 \mathrm{~A}$ ). Rate constants and the corresponding equilibrium binding constants $\left(K_{\mathrm{D}}\right)$ were calculated by applying a 1:1 Langmuir fit (Table 1). Figure 5B shows an overview, plotting one single concentration of each $\mathrm{R}$ subunit mutant protein interacting with immobilized $\mathrm{C}$ subunit. $\mathrm{R}$ A-domain WT and the proteins with Arg mutations displayed indistinguishable interaction kinetics (data not shown), as did $R$ A-domain Tyr229Phe (Figure 5B). R A-domain Leu203Trp showed fast association and fast dissociation, also reflected in a much lower equilibrium binding response. In contrast, R A-domain Tyr229Ala and Tyr229Thr had distinctly different binding patterns, displaying slower association and slower dissociation kinetics (see Table 1).

Again, the group of Arg proteins, probing the chargedcharged interactions, behaved like wild-type protein and could clearly be distinguished from the group of Tyr/Leu proteins, in which hydrophobic side chains were mutated, with the exception of R A-domain Tyr229Phe, which displayed wild-type-like interaction kinetics.

Since the $K_{d}$ values for holoenzyme formation showed huge differences, we also tested the dissociation behavior of preformed holoenzyme in response to cAMP using a spectrophotometric assay.

The activation constant $k_{\text {act }}$ (CAMP) for R A-domain WT holoenzyme of $1 \mu \mathrm{M}$ was in very good agreement with the previously published value $(1.1 \mu \mathrm{M}$; Ringheim and Taylor, 1990). Both proteins with mutations in Arg239 and Arg241, as well as Tyr229Phe, displayed wild-type-like behavior (data not shown). For all other R proteins with mutations of amino acids with hydrophobic side chains, activation constants could not be determined, since an extremely high excess of $R$ subunit was necessary to form an inhibitory $R$ subunit/C subunit complex.

cAMP binding to the isolated $R$ subunit was also investigated. A solution competition assay performed on a Biacore instrument was used to quantify cAMP binding (Zimmermann et al., 2002; D. Moll et al., in preparation; Table 2, Figure 6). R A-domain Arg239Ala/Glu, Arg 241Ala/Glu and Tyr229Phe displayed $E_{50}$ values in the same range as $\mathrm{R}$ A-domain WT (20-24 nM). R A-domain Tyr229Ala displayed a four-fold enhanced $\mathrm{EC}_{50}$ value. For Leu203Ala and Leu203Trp, the $\mathrm{EC}_{50}$ value was shifted by a factor of 35 and 120, respectively, into the micromolar range. The most striking difference was measured for $R$ A-domain Tyr229Asp. Here the cAMP concentration needed for half-maximal binding competition was $8.3 \mu \mathrm{M}$, i.e., 350-fold higher than for R A-domain WT.

Table 1 Association and dissociation rate constants for the $\mathrm{R}$ A-domain WT and mutants binding to immobilized $\mathrm{C} \alpha$ subunit using surface plasmon resonance.

\begin{tabular}{llcrl}
\hline R A-domain & \multicolumn{1}{c}{$\begin{array}{c}k_{\text {ass }} \times 10^{6} \\
\left(\mathrm{M}^{-1} \mathrm{~s}^{-1}\right)\end{array}$} & $\begin{array}{c}k_{\text {diss }} \times 10^{-3} \\
\left(\mathrm{~s}^{-1}\right)\end{array}$ & $\begin{array}{c}K_{\mathrm{d}} \\
(\mathrm{nM})\end{array}$ & $n$ \\
\hline WT & $0.69 \pm 0.63$ & $8.6 \pm 2.6$ & 12 & 4 \\
R239A & $1.1 \pm 0.2$ & $11 \pm 1.7$ & 10 & 3 \\
R239E & $1.4 \pm 0.55$ & $16 \pm 1.8$ & 11 & 3 \\
R241A & $0.47 \pm 0.088$ & $6.8 \pm 1.0$ & 14 & 3 \\
R241E & $1.3 \pm 0.36$ & $5.4 \pm 0.6$ & 4 & 3 \\
L203A & 0.029 & 8.2 & 283 & 1 \\
L203W & $0.094 \pm 0.06$ & $20 \pm 7.7$ & 212 & 3 \\
Y229A & $0.012 \pm 0.0041$ & $4.3 \pm 2.2$ & 358 & 3 \\
Y229F & $1.3 \pm 0.31$ & $25 \pm 2.7$ & 19 & 3 \\
Y229T & $0.06 \pm 0.022$ & $4.3 \pm 2.8$ & 72 & 6 \\
\hline
\end{tabular}

The association $\left(k_{\text {ass }}\right)$ and dissociation $\left(k_{\text {diss }}\right)$ rate constants are derived from curve fits with a 1:1 Langmuir binding model. The standard deviation is indicated. 
Again, two groups of proteins could clearly be distinguished. R A-domain WT and mutant proteins (Arg239Ala/Glu, Arg241Ala/Glu) with disrupted charged amino acids displayed similar cAMP-binding behavior. The protein containing the conservative mutation Tyr229Phe also belongs to this group. The other group of proteins involving changes in hydrophobic amino acids strongly lowered the affinity for cAMP binding. $R$ A-domain Tyr229Thr exhibited a 2.6-fold higher $\mathrm{EC}_{50}$ value, again ranging between the two groups.

\section{Discussion}

Sequence alignments of cAMP-binding proteins throughout the animal kingdom reveal a highly conserved region responsible for cAMP binding (Shabb and Corbin, 1992). Each cAMP-binding domain of the PKA $R$ subunits is composed of three $\alpha$-helices and an eight-stranded $\beta$ barrel (Su et al., 1995), forming a highly conserved phosphate binding cassette (PBC; Gly-Glu-Leu-AlaLeu- $\mathrm{X}_{3-5}$-Pro-Arg-Ala/Thr-Ala-Thr/Ser; Diller et al., 2001; Canaves and Taylor, 2002) responsible for phosphate binding and orientating the ribose moiety, and a nonconserved base binding region (BBR; Rehmann et al., 2003). The BBR contacts the base of the cyclic nucleotide and mediates site selectivity of CAMP analogs (Ogreid et al., 1989). Amino acids located in primary interaction sites with CAMP are conserved in all CAMPbinding domains and have been studied intensively (Houge et al., 1990; Neitzel et al., 1991; Herberg et al., 1996; Diller et al., 2001). Here we investigated the mode of action of cAMP on a second level of interaction, describing the molecular rearrangements ending in dissociation of the catalytic entity.

The mode of action of cAMP on the PKA holoenzyme is remarkably different from other CAMP-binding proteins. In contrast to Epac, in which a simple conformational switch results in catalytic GEF activity, in PKA the dissociation of two separate subunits is initiated by CAMP, involving several levels of molecular rearrangements.

By comparing the sequences of $R$ subunits, including non-vertebrate proteins, and other cyclic nucleotidebinding proteins (CAP, Epac, $\mathrm{K}^{+}$channels), several conserved amino acids outside the $\mathrm{PBC}$ can be identified. $\mathrm{A}$ mode of action of CAMP based on interaction of hydrophobic amino acids has already been proposed for Epac (Rehmann et al., 2003). These authors attributed binding of cAMP to a reorientation of invariant Leu and Tyr residues, which induces a small conformational change in the $\mathrm{PBC}$ region. This change is transmitted via a hinge into a large structural movement of a lid region (Diller et al., 2001) formed by the $\alpha$ C-helix (B/C helix; Kim et al., 2005), one of the flanking $\alpha$-helices that stabilizes the base. A lid mechanism has been discussed for the hyperpolarization-activated, cyclic nucleotide-modulated ( $\mathrm{HCN})$ pacemaker channels, in which the cyclic nucleotide-binding domain inhibits activation of the core transmembrane domain, and cAMP binding relieves this inhibition (Wainger et al., 2001). Using cysteine mutations and subsequent interaction analysis with sulfhydryl re-
Table 2 Competition assay for cAMP binding of the R Adomain using surface plasmon resonance.

\begin{tabular}{lcc}
\hline R A-domain & $\begin{array}{c}\text { cAMP EC } \\
(\mathrm{nM})\end{array}$ & $n$ \\
\hline WT & $24 \pm 5.4$ & 3 \\
R239A & $20 \pm 4.6$ & 3 \\
R239E & $21 \pm 3.3$ & 3 \\
R241A & $22 \pm 3.0$ & 2 \\
R241E & $21 \pm 0.4$ & 3 \\
L203A & $820 \pm 570$ & 7 \\
L203W & $2900 \pm 510$ & 4 \\
Y229A & $94 \pm 33$ & 7 \\
Y229D & $8300 \pm 4500$ & 3 \\
Y229F & $17 \pm 2.8$ & 3 \\
Y229T & $62 \pm 3.5$ & 3 \\
\hline
\end{tabular}

The $\mathrm{EC}_{50}$ values for $\mathrm{cAMP}$ were derived from surface plasmon resonance measurements on a $3 \mathrm{mM}$ 8-AHA-cAMP surface based on dose-response curves. The standard deviation is indicated.

agents, Mazzolini et al. (2002) could show movement of an $\alpha \mathrm{C}$-helix during $\mathrm{CNG}$ channel gating, also supporting a lid mechanism. A similar topology is found in the CAP protein (Weber and Steitz, 1987).

Leu273 (corresponding to Leu408 in Epac2) and Phe300 (Phe435 in Epac2) are conserved in Epac1. A Leu residue is also highly conserved in PKA $R$ subunits (Leu203 in bovine Rl $\alpha$ ). In PKA R subunits from all species analyzed so far, the Phe residue is also conserved, except for the A-domain of Rl $\alpha$ and RI $\beta$, in which the Phe is replaced by a Tyr residue (Tyr229 in bovine Rl $\alpha$; Shabb and Corbin, 1992; Canaves and Taylor, 2002; see Figure 1). The cyclic nucleotide-binding domains of CAP

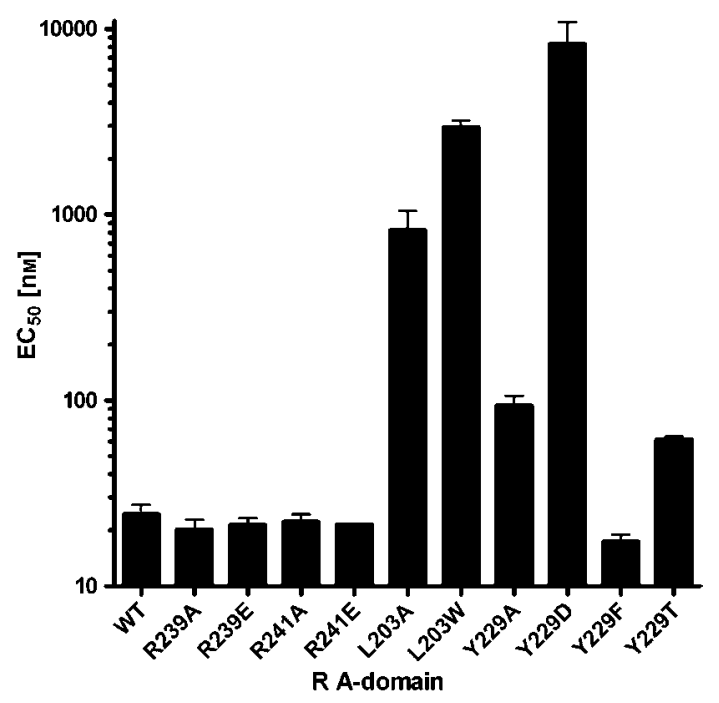

Figure $6 \quad \mathrm{EC}_{50}$ values for CAMP binding of the R A-domain WT and mutants measured with surface plasmon resonance (competition assay).

$R$ mutant proteins were incubated with different concentrations of cAMP and injected over a $3 \mathrm{~mm}$ 8-AHA-cAMP surface (see materials and methods). The response at the end of the association phase of the resulting binding curves was plotted against the cAMP concentration; $\mathrm{EC}_{50}$ values (see Table 2) were determined and are plotted here. Note the logarithmic scale of the $y-$ axis. 
in E. coli, the small GTPases Epac1 and Epac2 and the hyperpolarized $\mathrm{K}^{+}$channel also contain a Phe residue. Accordingly, probing this rather conserved amino acid change by mutating the critical Tyr229 to Phe in Rl $\alpha$ Adomain revealed no effect on any parameter tested.

cAMP binding and $R$ subunit/C subunit interaction need to be co-ordinated to allow dissociation of the PKA holoenzyme. In the PKA R A-domain, replacing Leu203 with Trp caused a 120-fold reduced $K_{d}$ for cAMP and a 10 -fold excess of mutant $R$ subunit was required to form holoenzyme. Since the substitution of Leu203 with Ala showed a similar effect on holoenzyme formation and a 35-fold reduced $K_{d}$ for cAMP binding (Table 2), the effect cannot be exclusively attributed to the bulky nature of Trp. Epac1 Leu273Trp (corresponding to Leu203Trp in R A-domain) can still bind cAMP with a five-fold reduced $K_{\mathrm{d}}$ (4 $\mu \mathrm{M}$ for WT, $20 \mu \mathrm{M}$ for Leu273Trp). However, in this case cAMP binding does not result in the activation of Epac1. Therefore, Rehmann et al. (2003) concluded that in Epac1 the binding and the conformational switch are uncoupled from each other.

In contrast, substitutions of Phe300 (corresponding to Tyr229 in R A-domain) with either Ala or Thr destabilize the ligand-free conformation of Epac1. Activation of Epac1 Phe300Ala and Phe300Thr was achieved at lower cAMP concentrations compared to the wild-type protein (Rehmann et al., 2003). Analysis of the corresponding mutant proteins R A-domain Tyr229Ala and Tyr229Thr resulted in $K_{d}$ values for cAMP with four- and 2.6-fold lower affinity, respectively, compared to R A-domain WT (Table 2). When Tyr229 is replaced by an Asp (R Adomain Tyr229Asp), severe effects on cAMP binding were observed, with a 350 -fold reduced affinity. This clearly demonstrates that in PKA, Leu203 and Tyr229 are crucial for transmitting cAMP binding to the $R$ subunit, as well as for maintaining $\mathrm{R} / \mathrm{C}$ subunit interaction.

The experiments presented here support a mechanism for CAMP action in PKA comparable to the activation mechanism of Epac (Rehmann et al., 2003). For the effects of binding of cAMP to the $R$ subunit and R/C interaction, the following model can be envisioned. If the concentration of CAMP is low, cAMP diffusing from the regulatory subunits will be replaced by water in the cyclic nucleotide-binding pocket, initiating hydrogen bonding to Glu200. Other water molecules are orientated accordingly and form a cap around the hydrophobic Leu201. The packing-induced optimal hydrogen-bonding distance of these capping water molecules induces a movement of Leu201, causing a shift of the backbone, which is further transmitted via Ala202 to Leu203 (Figure 7). Leu203 is part of a hydrophobic core region formed by Leu203, Ile204, Phe172, Tyr229 and Leu233 (Figure 8). The movement of the backbone around Leu203 induces a van-derWaals-exerted mechanical force to Tyr229, causing a rearrangement of the whole $\alpha \mathrm{C}$-helix, which in turn permits the $C$ subunit to access the $R$ subunit.

During the preparation of this manuscript, a high-resolution crystal structure of a complex between the C subunit and a deletion mutant of the $\mathrm{R}$ subunit (bovine Rl $\alpha \Delta 1-90 \Delta 245-379$, missing 15 amino acids at the Cterminal part compared to our R A-domain) was published (Kim et al., 2005). This structure confirms the data

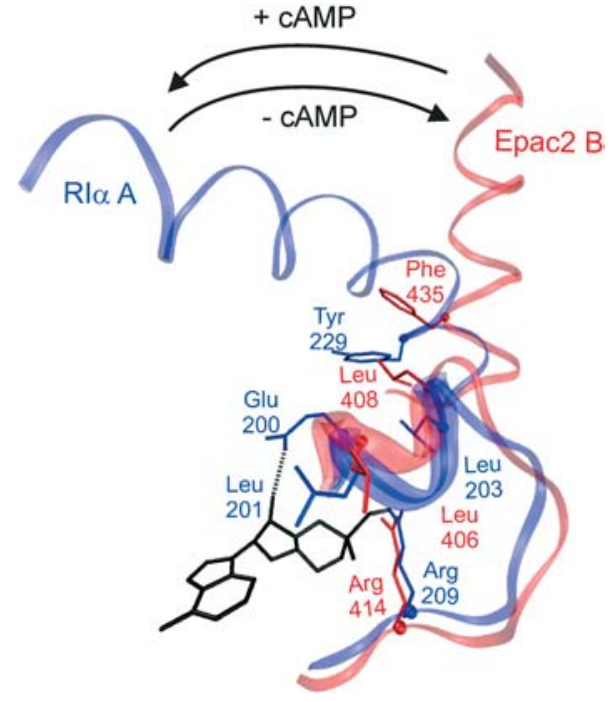

Figure 7 Overlay of the crystal structures of Rl $\alpha$ and Epac2. Alignment of the phosphate-binding cassette (PBC) and the adjacent helix of bovine Rl $\alpha$ A-domain (blue, protein data bank accession number 1NE4; Wu et al., 2004b) and murine Epac2 B-domain (red, 1O7F; Rehmann et al., 2003) (amino acids 196-215 and 401-420, respectively, were used for the alignment). Important residues are indicated for Rl $\alpha$ (Glu200, Leu201, Leu203, Arg209, Tyr229) and Epac2 (Leu406, Leu408, Arg414, Phe435). $R_{\mathrm{p}}$-adenosine- $3^{\prime}, 5^{\prime}$-cyclic monophosphorothioate $\left(R_{\mathrm{p}}\right.$ cAMPS, 1NE4; Wu et al., 2004b) is represented in black. For details see the text.

presented here, i.e., a hydrophobic region at the tip of the $\alpha \mathrm{C}$-helix seems to be important for conformational rearrangements. However, a crystal structure of the entire holoenzyme $\left(\mathrm{R}_{2} \mathrm{C}_{2}\right.$ complex) is needed to completely understand the activation mechanism of PKA at the molecular level. An $\mathrm{R}_{2} \mathrm{C}_{2}$ crystal structure may not only define the interactions between $\mathrm{R}$ and $\mathrm{C}$ subunits, but may also explain how the cyclic nucleotide-binding domains mediate cooperative binding of cAMP within the $\mathrm{R}$ dimer.

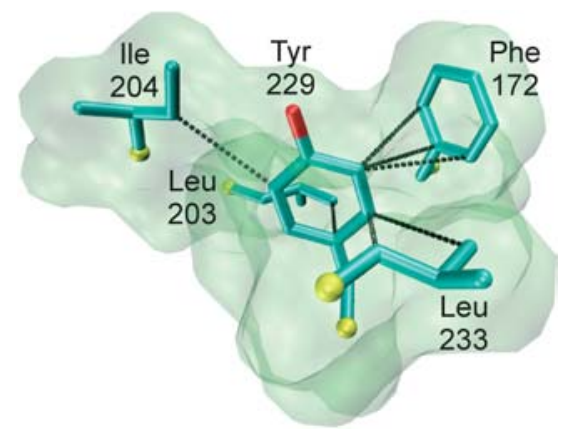

Figure 8 Hydrophobic core in the Rl $\alpha$ A-domain. Tyr229 represents the center of a hydrophobic core formed by the adjacent amino acids Leu203, Ile204, Phe172, and Leu233 (bovine Rl $\alpha$ A-domain, protein data bank accession number 1NE4; Wu et al., 2004b). The van-der-Waals' radii of the involved atoms are shown in a surface representation. 


\section{Materials and methods}

\section{Reagents}

The peptide substrate Kemptide (LRRASLG) was purchased from either Bachem AG (Bubendorf, Germany) or Biosyntan $\mathrm{GmbH}$ (Bremen, Germany). ATP and NADH were obtained from Biomol GmbH (Hamburg, Germany). cAMP (adenosine-3',5'cyclic monophosphate), 8-AHA-cAMP [8-(6-aminohexyl)aminoadenosine-3', $5^{\prime}$-cyclic monophosphate] and 6-AH-cAMP [N6-(6-aminohexyl)adenosine-3',5'-cyclic monophosphate] agarose were purchased from Biolog Life Science Institute (Bremen, Germany). Materials used in cloning were of molecular biology grade. Media supplies $\left(D_{i f c o}{ }^{T M}\right)$ were purchased from Becton Dickinson GmbH (Heidelberg, Germany). DNAs for the recombinant proteins were a kind gift from Prof. S.S. Taylor, University of California, San Diego, USA.

\section{Site-directed mutagenesis}

We used a construct of the isolated cAMP-binding domain A encoding the amino acids 93-259 of bovine Rl $\alpha$ (bovine Rl $\alpha$ $\Delta$ 1-92 $\Delta$ 260-379; Ringheim and Taylor, 1990; this is referred to as R A-domain). According to the QuikChange ${ }^{\circledR}$ Site-Directed Mutagenesis Kit protocol (Stratagene, La Jolla, USA), a pair of primers (MWG Biotech, Ebersberg, Germany) was used to introduce single amino acid exchanges in a single PCR. The following amino acid changes were made: Leu203Ala, Leu203Trp, Tyr229Ala, Tyr229Asp, Tyr229Thr, Tyr229Phe, Arg239Ala, Arg239Glu, Arg241Ala, and Arg241Glu. All mutations were confirmed by customer DNA sequence analysis performed by GATC Biotech AG, Konstanz, Germany.

\section{Expression of recombinant proteins}

Recombinant murine $\mathrm{C} \alpha$ was expressed and purified as described previously (Slice and Taylor, 1989; Herberg et al., 1993). After expression of recombinant $R$ subunits in $E$. coli BL21DE3 cells $\left(48 \mathrm{~h}, 37^{\circ} \mathrm{C}\right)$, the cells were harvested $(5000 \mathrm{~g}$, $15 \mathrm{~min}, 4^{\circ} \mathrm{C}$ ). The pellets were stored at $-20^{\circ} \mathrm{C}$.

\section{Purification of $\mathbf{R}$ subunits using cAMP resin}

Cells from $0.5 \mathrm{I}$ of culture were thawed on ice and resuspended in $15 \mathrm{ml}$ of lysis buffer $(20 \mathrm{~mm}$ potassium phosphate, $\mathrm{pH} 6.8$, $100 \mathrm{~mm} \mathrm{NaCl}, 2$ mм EDTA, 2 mm EGTA, 1 mM $\beta$-mercaptoethanol). The following steps were performed at $4^{\circ} \mathrm{C}$, unless stated otherwise. SDS-PAGE (Laemmli, 1970) checks were run subsequently. After homogenization, the homogenate was either passed through a French ${ }^{\circledR}$ Pressure Cell Press (Thermo Electron Corp., Needham Heights, USA) at 10 000-15000 psi or sonicated four times on ice with 30 pulses each $(0.7 \mathrm{~s}$ repeating duty cycle, circa 170-190 output, Labsonic U, B. Braun, Melsungen, Germany). The lysate was then centrifuged at $47800 \mathrm{~g}$ for $45 \mathrm{~min}$. The supernatant was rotated overnight (17-22 h) with $250 \mu \mathrm{l}$ of equilibrated 6-AH-cAMP agarose slurry. The agarose was pelleted $(450 \mathrm{~g}, 2 \mathrm{~min})$, transferred into a $1.5-\mathrm{ml}$ reaction tube and washed six times $(200 \mathrm{~g}, 2 \mathrm{~min})$ with $1 \mathrm{ml}$ of buffer A (20 mM MOPS, pH 7, $150 \mathrm{~mm} \mathrm{NaCl}, 1 \mathrm{~mm} \beta$-mercaptoethanol). Proteins were eluted with $1 \mathrm{ml}$ of $10 \mathrm{mM}$ cGMP at room temperature $\left(22^{\circ} \mathrm{C}\right)$ for $1 \mathrm{~h}$. The supernatant was passed over a NAP10 gel filtration column (Amersham Pharmacia Biotech, Freiburg, Germany) equilibrated in buffer A. The protein was eluted with $1.5 \mathrm{ml}$ of buffer $A$ and dialyzed overnight against buffer $A$ (three buffer exchanges). The proteins (R A-domain) were then either stored on ice or at $-20^{\circ} \mathrm{C}$ for further use.

\section{Circular dichroism (CD) measurements}

Each protein was exchanged to $5 \mathrm{~mm}$ potassium phosphate, $\mathrm{pH} 7$ on a Superose 12 column pre-equilibrated in the same buffer using an FPLC system (Amersham Biosciences). CD measurements were performed on a Jasco J-720 spectropolarimeter using $15 \mu \mathrm{M}$ protein in a $0.1-\mathrm{cm}$-pathlength cuvette (300 $\mu$ l capacity) at $22^{\circ} \mathrm{C}$. CD spectra were scanned from 195 to $255 \mathrm{~nm}$ at a scanning speed of $50 \mathrm{~nm} / \mathrm{min}$ with $0.5-\mathrm{nm}$ data pitch and an integration time of $2 \mathrm{~s}$. From two independent preparations, 20 spectra were accumulated for each protein. The baseline was recorded for buffer only and subtracted. Conversion of observed ellipticity values (mdeg) to mean residue ellipticity (deg $\mathrm{cm}^{2} / \mathrm{dmol}$ ) was carried out according to the equation:

$[\Theta]_{\mathrm{MRW}}=\frac{\Theta \cdot 100 \cdot M_{\mathrm{r}}}{c \cdot 1 \cdot N_{\mathrm{A}}}$

where $\Theta$ is the measured ellipticity (deg), $c$ the protein concentration $(\mathrm{mg} / \mathrm{ml}), I$ is the pathlength $(\mathrm{cm}), M_{\mathrm{r}}$ is the protein molecular weight and $N_{\mathrm{A}}$ is the number of amino acids in the respective protein.

\section{Holoenzyme formation}

A fixed $C$ subunit concentration (20-30 nM) was incubated for 1 min with varying concentrations of $R$ subunit in $100 \mu$ l of assay mix at room temperature $\left(22^{\circ} \mathrm{C}\right)$ according to Cook et al. (1982). The reaction was started by addition of the heptapeptide Kemptide (200 $\mu \mathrm{M}$ final concentration) and followed for $1 \mathrm{~min}$ at $340 \mathrm{~nm}$ using a spectrophotometer (Lambda Bio UV/Vis Spectrometer, Perkin Elmer, Rodgau-Jügesheim, Germany, or Specord 205, Analytik Jena, Jena, Germany). $\mathrm{EC}_{50}$ values were extracted from sigmoidal dose-response curves using GraphPad Prism 4.02. The ability to dissociate holoenzyme was checked by the addition of $75 \mu \mathrm{M}$ cAMP.

\section{Surface plasmon resonance}

Biacore 2000 or Biacore 3000 instruments (Biacore AB, Uppsala, Sweden) were used to study the interaction of free $\mathrm{R} A$-domain WT and mutants with either immobilized $\mathrm{C} \alpha$ subunit or immobilized cAMP analogs.

Three different $C$ subunit preparations were immobilized on a CM 5 sensor chip (Biacore AB) using amine coupling (Herberg et al., 1994; Hahnefeld et al., 2004). The $C$ subunits were diluted to $10-30 \mu \mathrm{g} / \mathrm{ml}$ in $10 \mathrm{~mm}$ sodium acetate, $500 \mu \mathrm{M}$ ATP, $1 \mathrm{~mm}$ $\mathrm{MgCl}_{2}$ with a pH between 5.5 and 6.0. Then 470 response units ( $\mathrm{RU}$; $1000 \mathrm{RU}=1 \mathrm{ng} / \mathrm{mm}^{2}$; Stenberg et al., 1991) were immobilized as described previously (Herberg et al., 1994). A reference surface was activated/deactivated without protein, and the response was subtracted. All interaction experiments with $R$ subunit were performed at $25^{\circ} \mathrm{C}$ in running buffer $(20 \mathrm{~mm}$ MOPS, $\mathrm{pH} 7,150 \mathrm{~mm} \mathrm{NaCl}, 500 \mu \mathrm{M}$ ATP, $1 \mathrm{~mm} \mathrm{MgCl}_{2}, 0.005 \%$ surfactant P20) at a flow rate of $20 \mu \mathrm{l} / \mathrm{min}$. After simultaneous R subunit injection over the four surfaces ( $3 \mathrm{~min}$ ), the dissociation phase was monitored for $1 \mathrm{~min}$. The binding of $R$ subunit to $C$ subunit was not limited by mass transport, as demonstrated previously (Herberg and Zimmermann, 1999). Sensor surfaces were regenerated by two subsequent injections (1 min each) with $1 \mathrm{mM}$ CAMP, 2 mM EDTA in running buffer. Data evaluation was performed using Biaevaluation 4.1 (Biacore $A B$ ) and a 1:1 Langmuir binding model was applied for the $C$ subunit/R subunit interaction (Hahnefeld et al., 2004).

Protein/nucleotide interaction was monitored using a solution competition assay (Zimmermann et al., 2002; D. Moll et al., in preparation). In brief, R subunits (10 or $50 \mathrm{~nm}$ ) were preincubated (30 min) with varying amounts of free cAMP and subsequently 
injected over a $3 \mathrm{~mm}$ 8-AHA-cAMP surface. The response at the end of the dissociation phase ( $t=120 \mathrm{~s}$ ) was plotted against the free cAMP concentration and $\mathrm{EC}_{50}$ values were calculated with GraphPad Prism.

\section{Structure-based alignments}

Structure-based alignments were performed using the X-ray crystal structures of bovine Rl $\alpha$ A-domain (protein data bank accession number 1NE4; Wu et al., 2004b) and murine Epac2 B-domain (107F; Rehmann et al., 2003). The alignment was adjusted on the PBC (Canaves and Taylor, 2002) region (bovine Rl $\alpha$, amino acids 196-215; murine Epac2, amino acids 401-420) using XtalView 4.0/XFit 4.1 (McRee, 1999) and illustrated with Visual Molecular Dynamics 1.8.2 (VMD; Humphrey et al., 1996).

\section{Acknowledgments}

We thank Dr. Silke Hutschenreiter (University of Kassel, Germany), Prof. Susan Taylor (University of San Diego, USA), Prof. Alfred Wittinghofer and Dr. Holger Rehmann (MPI, Dortmund, Germany), and Prof. Hartmut Follmann and Prof. Wolfgang Nellen (University of Kassel, Germany) for helpful discussions. We are grateful to Sonja Schweinsberg, Nicole Burghardt and Elke Böhm (University of Kassel, Germany) for superb technical help and would like to thank Dr. Wolfgang Hoyer (MPI for Biophysical Chemistry, Göttingen, Germany) and Dennis Fiegen (MPI, Dortmund, Germany) for help with the CD experiments. This work was supported by grants from the Deutsche Forschungsgemeinschaft (DFG, He1818/4), the European Commission (EUCRAFT QLK2-CT-2002-72419), and the Bundesministerium für Bildung und Forschung (BMBF, 01 GR 0441) to F.W. Herberg.

\section{References}

Anand, G.S., Hughes, C.A., Jones, J.M., Taylor, S.S., and Komives, E.A. (2002). Amide $\mathrm{H} /{ }^{2} \mathrm{H}$ exchange reveals communication between the CAMP and catalytic subunit-binding sites in the $\mathrm{R}(\mathrm{I}) \alpha$ subunit of protein kinase A. J. Mol. Biol. 323, 377-386.

Bossemeyer, D., Engh, R.A., Kinzel, V., Ponstingl, H., and Huber, R. (1993). Phosphotransferase and substrate binding mechanism of the CAMP-dependent protein kinase catalytic subunit from porcine heart as deduced from the $20 \AA$ structure of the complex with $\mathrm{Mn}^{2+}$ adenylyl imidodiphosphate and inhibitor peptide PKI(5-24). EMBO J. 12, 849-859.

Buechler, J.A. and Taylor, S.S. (1990). Differential labeling of the catalytic subunit of CAMP-dependent protein kinase with a water-soluble carbodiimide: identification of carboxyl groups protected by MgATP and inhibitor peptides. Biochemistry 29, 1937-1943.

Canaves, J.M. and Taylor, S.S. (2002). Classification and phylogenetic analysis of the cAMP-dependent protein kinase regulatory subunit family. J. Mol. Evol. 54, 17-29.

Cook, P.F., Neville, M.E. Jr., Vrana, K.E., Hartl, F.T., and Roskoski, R. Jr. (1982). Adenosine cyclic 3',5'-monophosphate dependent protein kinase: kinetic mechanism for the bovine skeletal muscle catalytic subunit. Biochemistry 21, 5794-5799.

de Rooij, J., Zwartkruis, F.J., Verheijen, M.H., Cool, R.H., Nijman, S.M., Wittinghofer, A., and Bos, J.L. (1998). Epac is a Rap1 guanine-nucleotide-exchange factor directly activated by cyclic AMP. Nature 396, 474-477.

de Rooij, J., Rehmann, H., van Triest, M., Cool, R.H., Wittinghofer, A., and Bos, J.L. (2000). Mechanism of regulation of the Epac family of cAMP-dependent RapGEFs. J. Biol. Chem. 275, 20829-20836.
Diller, T.C., Madhusudan, Xuong, N.H., and Taylor, S.S. (2001). Molecular basis for regulatory subunit diversity in CAMPdependent protein kinase: crystal structure of the type II $\beta$ regulatory subunit. Structure (Camb.) 9, 73-82.

Gibson, R.M., Ji-Buechler, Y., and Taylor, S.S. (1997). Interaction of the regulatory and catalytic subunits of cAMP-dependent protein kinase. Electrostatic sites on the type l $\alpha$ regulatory subunit. J. Biol. Chem. 272, 16343-16350.

Hahnefeld, C., Drewianka, S., and Herberg, F.W. (2004). Determination of kinetic data using surface plasmon resonance biosensors. Methods Mol. Med. 94, 299-320.

Herberg, F.W. and Zimmermann, B. (1999). Analysis of protein kinase interactions using biomolecular interaction analysis. In: Protein Phosphorylation - A Practical Approach, Vol. 2, D.G. Hardie, ed. (Oxford, UK: Oxford University Press), pp. 335-371.

Herberg, F.W., Bell, S.M., and Taylor, S.S. (1993). Expression of the catalytic subunit of CAMP-dependent protein kinase in Escherichia coli: multiple isozymes reflect different phosphorylation states. Protein Eng. 6, 771-777.

Herberg, F.W., Dostmann, W.R., Zorn, M., Davis, S.J., and Taylor, S.S. (1994). Crosstalk between domains in the regulatory subunit of CAMP-dependent protein kinase: influence of amino terminus on CAMP binding and holoenzyme formation. Biochemistry 33, 7485-7494.

Herberg, F.W., Taylor, S.S., and Dostmann, W.R. (1996). Active site mutations define the pathway for the cooperative activation of cAMP-dependent protein kinase. Biochemistry 35 , 2934-2942.

Houge, G., Steinberg, R.A., Ogreid, D., and Doskeland, S.O. (1990). The rate of recombination of the subunits (RI and C) of cAMP-dependent protein kinase depends on whether one or two cAMP molecules are bound per RI monomer. J. Biol. Chem. 265, 19507-19516.

Huang, L.J. and Taylor, S.S. (1998). Dissecting cAMP binding domain $A$ in the Rl $\alpha$ subunit of CAMP-dependent protein kinase. Distinct subsites for recognition of CAMP and the catalytic subunit. J. Biol. Chem. 273, 26739-26746.

Humphrey, W., Dalke, A., and Schulten, K. (1996). VMD: visual molecular dynamics. J. Mol. Graph. 14, 27-38.

Kaupp, U.B. and Seifert, R. (2002). Cyclic nucleotide-gated ion channels. Physiol. Rev. 82, 769-824.

Kawasaki, H., Springett, G.M., Mochizuki, N., Toki, S., Nakaya, M., Matsuda, M., Housman, D.E., and Graybiel, A.M. (1998). A family of cAMP-binding proteins that directly activate Rap1. Science 282, 2275-2279.

Kim, C., Xuong, N.-H., and Taylor, S.S. (2005). Crystal structure of a complex between the catalytic and regulatory $(\mathrm{Rl} \alpha)$ subunits of PKA. Science 307, 690-696.

Knighton, D.R., Zheng, J.H., Ten Eyck, L.F., Xuong, N.H., Taylor, S.S., and Sowadski, J.M. (1991). Structure of a peptide inhibitor bound to the catalytic subunit of cyclic adenosine monophosphate-dependent protein kinase. Science 253, 414-420.

Krebs, E.G. and Beavo, J.A. (1979). Phosphorylation-dephosphorylation of enzymes. Annu. Rev. Biochem. 48, 923-959.

Laemmli, U.K. (1970). Cleavage of structural proteins during the assembly of the head of bacteriophage T4. Nature 227, 680-685.

Leon, D.A., Canaves, J.M., and Taylor, S.S. (2000). Probing the multidomain structure of the type I regulatory subunit of cAMP-dependent protein kinase using mutational analysis: role and environment of endogenous tryptophans. Biochemistry $39,5662-5671$.

Mazzolini, M., Punta, M., and Torre, V. (2002). Movement of the $\mathrm{C}$-helix during the gating of cyclic nucleotide-gated channels. Biophys. J. 83, 3283-3295.

McRee, D.E. (1999). XtalView/Xfit - A versatile program for manipulating atomic coordinates and electron density. J. Struct. Biol. 125, 156-165.

Morgenstern, B. (1999). DIALIGN 2: improvement of the segment-to-segment approach to multiple sequence alignment. Bioinformatics 15, 211-218. 
Neitzel, J.J., Dostmann, W.R., and Taylor, S.S. (1991). Role of MgATP in the activation and reassociation of cAMP-dependent protein kinase I: consequences of replacing the essential arginine in cAMP binding site A. Biochemistry 30, 733-739.

Ogreid, D. and Doskeland, S.O. (1981). The kinetics of the interaction between cyclic AMP and the regulatory moiety of protein kinase II. Evidence for interaction between the binding sites for cyclic AMP. FEBS Lett. 129, 282-286.

Ogreid, D., Ekanger, R., Suva, R.H., Miller, J.P., and Doskeland, S.O. (1989). Comparison of the two classes of binding sites (A and B) of type I and type II cyclic-AMP-dependent protein kinases by using cyclic nucleotide analogs. Eur. J. Biochem. 181, 19-31.

Rehmann, H., Prakash, B., Wolf, E., Rueppel, A., De Rooij, J., Bos, J.L., and Wittinghofer, A. (2003). Structure and regulation of the cAMP-binding domains of Epac2. Nat. Struct. Biol. 10, 26-32.

Ringheim, G.E. and Taylor, S.S. (1990). Dissecting the domain structure of the regulatory subunit of cAMP-dependent protein kinase I and elucidating the role of MgATP. J. Biol. Chem. 265, 4800-4808.

Shabb, J.B. and Corbin, J.D. (1992). Cyclic nucleotide-binding domains in proteins having diverse functions. J. Biol. Chem. 267, 5723-5726.

Slice, L.W. and Taylor, S.S. (1989). Expression of the catalytic subunit of cAMP-dependent protein kinase in Escherichia coli. J. Biol. Chem. 264, 20940-20946.

Sorol, M.R., Pastori, R.L., Muro, A., Moreno, S., and Rossi, S. (2000). Structural and functional analysis of the cAMP binding domain from the regulatory subunit of Mucor rouxii protein kinase A. Arch. Biochem. Biophys. 382, 173-181.

Stenberg, E., Persson, B., Roos, H., and Urbaniczky, C. (1991). Quantitative determination of surface concentration of protein with surface plasmon resonance using radiolabeled proteins. J. Colloid Interface Sci. 143, 513-526.

Stergiopoulos, S.G. and Stratakis, C.A. (2003). Human tumors associated with Carney complex and germline PRKAR1A mutations: a protein kinase A disease! FEBS Lett. 546, 59-64.
Su, Y., Dostmann, W., Herberg, F.W., Durick, K., Xuong, N.H., Ten Eyck, L., Taylor, S.S., and Varughese, K.I. (1995). Regulatory subunit of protein kinase $A$ : structure of a deletion mutant with cAMP binding domains. Science 269, 807-813.

Symcox, M.M., Cauthron, R.D., Ogreid, D., and Steinberg, R.A. (1994). Arg-242 is necessary for allosteric coupling of cyclic AMP-binding sites A and B of RI subunit of cyclic AMPdependent protein kinase. J. Biol. Chem. 269, 23025-23031.

Tasken, K. and Aandahl, E.M. (2004). Localized effects of cAMP mediated by distinct routes of protein kinase A. Physiol. Rev. 84, 137-167.

Taylor, S.S., Buechler, J.A., and Yonemoto, W. (1990). cAMPdependent protein kinase: framework for a diverse family of regulatory enzymes. Annu. Rev. Biochem. 59, 971-1005.

Wainger, B.J., DeGennaro, M., Santoro, B., Siegelbaum, S.A., and Tibbs, G.R. (2001). Molecular mechanism of cAMP modulation of HCN pacemaker channels. Nature 411, 805-810.

Weber, I.T. and Steitz, T.A. (1987). Structure of a complex of catabolite gene activator protein and cyclic AMP refined at $2.5 \AA$ resolution. J. Mol. Biol. 198, 311-326.

Weber, I.T., Steitz, T.A., Bubis, J., and Taylor, S.S. (1987). Predicted structures of cAMP binding domains of type I and II regulatory subunits of CAMP-dependent protein kinase. Biochemistry 26, 343-351.

Wu, J., Brown, S., Xuong, N.H., and Taylor, S.S. (2004a). Rl $\alpha$ subunit of PKA; a cAMP-free structure reveals a hydrophobic capping mechanism for docking CAMP into site B. Structure (Camb.) 12, 1056-1064.

Wu, J., Jones, J.M., Xuong, N.H., Eyck, L.F., and Taylor, S.S. (2004b). Crystal structures of Rl $\alpha$ subunit of cyclic adenosine $5^{\prime}$-monophosphate (CAMP)-dependent protein kinase complexed with $\left(R_{\mathrm{p}}\right)$-adenosine $3^{\prime}, 5^{\prime}$-cyclic monophosphothioate and $\left(S_{\mathrm{p}}\right)$-adenosine $3^{\prime}, 5^{\prime}$-cyclic monophosphothioate, the phosphothioate analogues of cAMP. Biochemistry 43 , 6620-6629.

Zimmermann, B., Hahnefeld, C., and Herberg, F.W. (2002). Applications of biomolecular interaction analysis in drug development. TARGETS 1, 66-73.

Received February 9, 2005; accepted May 10, 2005 